\title{
Optimization of Immune Receptor-Related Hypersensitive Cell Death Response Assay Using Agrobacterium-Mediated Transient Expression in Tobacco Plants
}

\section{Sung Un Huh ( $\nabla$ sungun8550@gmail.com )}

Kunsan National University https://orcid.org/0000-0003-2288-9579

\section{Research Article}

Keywords: NLR-type immune receptor, Pathogen avirulence effector, Cell death, Paired NLR, Tobacco, Agrobacterium, Hypersensitive response

Posted Date: February 8th, 2022

DOI: https://doi.org/10.21203/rs.3.rs-1307980/v1

License: (c) (1) This work is licensed under a Creative Commons Attribution 4.0 International License. Read Full License 


\section{Abstract}

\section{Background}

The study of the regulatory mechanisms of evolutionarily conserved Nucleotide-binding leucine-rich repeat (NLR) resistance (R) proteins in animals and plants is of increasing importance due to the understanding of basic immunity and the value of various crop engineering applications of NLR immune receptors. The importance of temperature is also emerging when applying NLR to crops responding to global climate change. In particular, studies of pathogen effector recognition and autoimmune activity of NLRs in plants can quickly and easily determine their function in tobacco using agro-mediated transient assay. However, there are conditions that should not be overlooked in these cell death-related assays in tobacco.

\section{Results}

Environmental conditions play an important role in the immune response of plants. The system used in this study was to establish conditions for optimal hypertensive response (HR) cell death analysis by utilizing the paired NLR RPS/RRS1 autoimmune and AvrRps4 effector recognition system. The most suitable greenhouse temperature for growing plants was fixed at $22^{\circ} \mathrm{C}$. In this study, RPS4/RRS1mediated autoimmune activity, RPS4 TIR domain dependent cell death, and RPS4/RRS1-mediated HR cell death upon AvrRps4 perception significantly inhibited under conditions of $65 \%$ humidity. The HR is strongly activated when the humidity is below $10 \%$. In addition, the leaf position of tobacco is important for the HR cell death. Position \#4 of the leaf from the top in 4-5 weeks old tobacco plants showed the most effective HR cell death.

\section{Conclusions}

As genome sequencing of various crops continues, different types of NLRs and their functions will be studied. At this time, if we optimize the conditions for evaluating NLR-mediated HR cell death, it will help to more accurately identify the function of NLRs. In addition, it will be possible to contribute to crop development in response to global climate change through NLR engineering.

\section{Introduction}

Recent climate change is changing the map of crop production. An increase in temperature can aggravate crop damage caused by pathogens ${ }^{1,2}$. In order to actively respond to these rapid changes, rapid response through genetic engineering is required.

Plant pathogens inject effector proteins by manipulating cellular processes via type three secreted system (T3SEs) into host cells ${ }^{3}$. Nucleotide-binding leucine-rich repeat receptor (NLR) proteins recognize specific effectors and trigger effector-triggered immunity (ETI), which is often associated with localized programmed cell death, known as the hypersensitive response (HR) and limits pathogen proliferation ${ }^{4}$. 
Plant NLRs share many similar structural features to animals ${ }^{5}$. How plant NLRs signal immune responses remains largely unknown when compared to similar animal systems.

Like animals, paired NLRs exist in plants, and their functions are divided into sensor NLR and helper NLR. In particular, sensor NLRs have a role in recognizing effectors via integrated domain and inhibit the autoimmune activity of helper NLRs ${ }^{6}$. For example, this is the case with RPS4/RRS1. RRS1 is known to recognize effectors secreted by three different pathogens 7,8 . Interestingly, artificially extending the $\mathrm{N}$ - or C-terminus of RRS1 activates RPS4 autoimmunity without effector recognition ${ }^{9}$. That is, an autoimmune system and an effector recognition system using RPS4/RRS1 paired NLR can be studied together.

Agrobacterium-mediated transient analysis is widely used to evaluate the function of NLRs. This is because the clear phenotype of HR cell death can be assessed with various combinations of NLRs and effectors in a relatively short time without obtaining the transgenic plants. It is known that NLR-mediated cell death is influenced by environmental factors such as temperature and humidity 10,11 . In particular, the immunity of plants decreases with increasing temperature conditions and affects effector recognition of NLRs ${ }^{12}$. If so, it is important to clarify the conditions for an effective HR cell death assay using agrobacterium-mediated transient system in tobacco plant.

In this study, we confirmed that humidity and plant leaf position, excluding temperature conditions, were important for effectively confirming HR cell death assay in tobacco plant. The RPS4/RRS1-mediated cell death was activated by AvrRps4 perception in tobacco plants, these HR cell death was suppressed at $65 \%$ humidity. However, in 10\% humidity, the HR cell death was enhanced. In addition, the infiltrated-leaf position of tobacco is important. Position \#4 of recent leaves from the tops in 4-5 weeks old plants showed the most effective HR cell death. Under these distinct conditions, performing NLR-mediated HR upon effector recognition or autoimmune activation may yield more accurate results.

\section{Results}

The NLR RPS4/RRS1 pair can be used in the cell death assay system, which is divided into autoimmune activity and effector-triggerred hypersensitive cell death response

Two NLR immune receptor proteins encoded by genetically linked genes function together as paired NLRs. They are divided into sensor NLR and helper NLR according to their function. Sensor NLR frequently has a non-canonical domain called the integrated domain (ID) which can recognize cognate effector protein ${ }^{13,14}$. Overexpression of helper NLR caused HR-like cell death in absence of sensor NLR. If coexpressed sensor/helper NLRs, the HR cell death is inhibited (Figure 1A) ${ }^{13}, 15$. When a protein such as fluorescent protein was linked to the N-terminus or C-terminus of the sensor NLR RRS1, RPS4dependent autoimmunity appeared without effector perception (Figure 1A) ${ }^{9}$. In the engineering of sensor NLRs, it must be careful when engineering to utilize specific proteins. TIR (Toll-like, Interleukin-1 receptor, Resistance protein) or CC (coiled-coil) of the helper NLR act as a signaling domain. TIR ${ }^{\text {RPS4 }}$ domain alone can activate TIR-dependent autoimmune and TIR RRS1 can suppress this HR cell death ${ }^{16}$. TIR domain 
mutant RPS4(SH/AA) fail to activate HR, suggesting that TIR ${ }^{\mathrm{RPS} 4}$ oligomerization is required for generating the HR signal (Figure 1B). RRS1-R from accessions Ws-2 forms an immune receptor complex with RPS4 that recognizes Pseudomonas syringae AvrRps4, Ralstonia solanacearum PopP2, and unknown effector $7,8,17,18$. Thus the paired RPS4/RRS1-R can prevent infection by three distinct pathogens and it can be used as a very useful tool for crop engineering (Figure 1C). We summarize the following experimental procedures based on the RPS4/RRS1 system capable of performing these three types of HR cell death assays using the Agrobacterium-mediated transient expression in tobacco plant (Figure 1D).

\section{Both RRS1-S and RRS1-R fused with fluorescent protein under low humidity conditions exhibited RPS4- dependent HR cell death}

Sensor NLR is a good material for developing crops that recognize various pathogens by introducing new IDs. The positioning may alter the overall sensor NLR protein size or fuse new IDs into the N- or Cterminus ${ }^{19}$. Bimolecular fluorescence complementation (BiFC) methods to confirm intra-/intercellular interactions should evaluate their function when applied to sensor NLRs ${ }^{9}, 20$. We found that overexpression of cCFP-RRS1-S-nCerulean activated RPS4-depenent HR cell death in low humidity condition but this cell death was suppressed in the high humidity condition (Figure 2A). At 4 dpi, we could not detect any difference between CCFP-RRS1-S-nCerulean/RPS4-HA mediated autoimmune activity and cCFP-RRS1-S-nCerulean/RPS4-HA mediated HR cell death upon AvrRps4 perception (Figure 2A). As the result, it was confirmed that the humidity condition at a temperature of $22^{\circ} \mathrm{C}$ affected HR cell death.

In the case of RRS1-R, unlike RRS1-S, 83 amino acids are extended the C-terminus, which is known to play a decisive role in recognizing PopP2 ${ }^{21}$. We tested changes in autoimmunity under difference humidity conditions using RRS1R-cCFP and RRS1-R-nCerulean. Consistent with cCFP-RRS1-S-nCerulean, both RRS1-R-cCFP and RRS1-R-nCerulean activated RPS4-dependent HR cell death under low humidity. High humidity can suppress these cell death in N. tabaccum (Figure 2B). Finally, humidity is an important determinant of HR cell death activation by paired NLR autoimmune.

\section{Agro-infiltrated leaf location in the tobacco plant is also a critical factor in HR cell death assays}

HR cell death assays using tobacco plants often show differences in the intensities of HR cell death. In particular, if agro-infiltration is performed using various leaves in one plant, the same cell death cannot be obtained. To confirm that these differences occurred, leaf positions of 4-5 week old $N$. benthamiana were numbered from top to bottom. In the leaf position \#4, coexpression of RRS1-R-CCFP/RPS4-Myc or RRS1$R$-nCerulean/ RPS4-Myc exhibited strong HR cell death but not leaf position \#5 (Figure 3A). Consistently, this autoimmune activity was suppressed by high humidity in $N$. benthamiana (Figure 3B). These results suggested that HR cell death is also affected by infiltrated leaf position and can be more easily detected under a relatively low humidity condition. 
Agro-infiltrating leaf position is an important factor to evaluate HR cell death in both TIR RPS4 -mediated cell death and RPS4/RRS1-mediated effector triggered cell death

NLRs are divided into two groups depending on the type of N-terminal domain. The N-terminal coiled-coil (CC) domain is called CNLs (CC-NLRs), and those with the N-terminal Toll/interleukin-1 receptor (TIR) domain is called TNLs (TIR-NLRs) ${ }^{22}$. Evolutionally, bacterial TIR domain proteins have NADase enzymatic activity that generates a non-canonical variant cyclic ADPR (CADPR) molecule and cleave $\mathrm{NAD}^{+}$(nicotinamide adenine dinucleotide) ${ }^{23}$. The TIR domain of plant NLR also has NADase activity and is required downstream signaling 24,25 . The overexpression of TIR ${ }^{R P S 4}$ domain is sufficient to activate autoimmune and oligomerization of $\mathrm{TIR}^{\mathrm{RPS} 4}$ domain is required for plant immune signaling ${ }^{16}$. In the $\mathrm{TIR}^{\mathrm{R}}$ mediated immunity studies, the importance of TIR function evaluation is increasing.

The $N$. benthamiana is an excellent system for studying cell death induced by the TIR/CC domains. To test whether TIR ${ }^{R P S 4}$ can induce HR cell death in $N$. benthamiana and which leaf positions are most suitable for HR cell death assays. When co-delivered 35S::TIR RPS4-GFP/35S::GFP control in leaf positions \#4-\#7, the HR cell death is detected in leaf position \#4 and \#5 (Figure 4A). In the leaf position \#4, TIR RPS4mediated autoimmune cell death is more potent than leaf position \#5 (Figure 4A). In leaf positions \#6 and \#7, HR cell death is not detected (Figure 4A). If co-expressed 35S:::TIR ${ }^{R P S 4(S H / A A)}$-GFP/35S:::GFP control, It cannot induce HR cell death because of inhibition of TIR ${ }^{R P S 4}$ oligomerization (Figure 4A).

We also checked whether RPS4/RRS1 is functional in N. benthamiana system. When co-delivered 35S::RRS1-R-HF/35S:::RPS4-Myc/35S:::AvrRps4-GFP, clear HR cell death was observed in the leaf positions \#4, but weak HR cell death was exhibited in the leaf positions \#5 (Figure 4B). However, RPS4/RRS1mediated HR cell death upon AvrRps4 perception is not observed in leaf positions \#6-\#7 (Figure 4B). We attempted to determine whether HR intensity was determined by leaf position in $N$. tabacum as in the $N$. benthamiana. Similarly, combination of 35S::RRS1-R-HF/35S:::RPS4-Myc/35S::AvrRps4-GFP was coexpressed at leaf positions \#4-\#5 in $N$. tabacum. HR cell death phenotype was checked at $4 \mathrm{dpi}$. As expected, HR cell death was stronger in leaf position \#4 than \#5 (Figure 4C). In the effector triggered HR cell death, the position of the leaf used for Agro-infiltration affects the intensity of HR cell death both $N$. benthamiana and $N$. tabacum.

Based on the results, we have summarized the important factors in the tobacco-based Agro-infiltration cell death assay (Figure 5). In most reported cases, high temperature suppressed HR as well as disease resistance. The high temperature suppressed plant resistance under various experimental conditions ${ }^{10}$, ${ }^{11}$. In the HR cell death analysis, humidity and leaf position used for infiltration were found to be the most important factors. Ultimately, it is expected to be of great help in experiments to confirm the cell death phenotype that exhibits various NLRs under these optimal conditions. In addition, when preparing a sample for co-immunoprecipitation (co-IP) or western blot analysis rather than a cell death assay, if the humidity is high and the sample is collected from a location other than location \#4, sufficient experiments are possible. 


\section{Discussion}

In this study, we developed and optimized an Agro-mediated transient cell death assay in tobacco plant. Tobacco plants are the most effective functional research system as various types of plant NLRs have been discovered through whole genome sequencing (WGS) $26,27,28$. In particular, it is possible to quickly and easily identify which effector NLR recognizes or which domain regulates NLR autoimmune activity through phenotypes such as HR cell death. On the other hand, it is difficult to confirm the intracellular localization of the NLR fused with a fluorescent protein due to autoimmune activity. Some NLRs do not produce distinct HR responses in tobacco plant. That is, updated low-humidity conditions and infiltrated leaf positions can be utilized to identify optimal HR cell death phenotypes of NLR studies.

Regulation mechanism of NLR-mediated humidity-sensitive HR is not fully understood. However, this is probably similar to the temperature-sensitive HR cell death. Although there are exceptions and not much research has been done, high temperature and humidity suppressed HR and affected plant immunity ${ }^{10}$, $11,29,30$. For example, dwarf phenotype of autoimmune snc1-1 is suppressed at $28^{\circ} \mathrm{C}^{31}$. Arabidopsis Ubox ubiquitin ligase SAUL1 regulated senescence and cell death. The saul1-1 mutant showed the autoimmune activity. An autoimmune phenotype of saul1-1 was rescued by higher relative humidity and higher temperature. The saul1-1 phenotype regulated by EDS1/PAD4 dependent signaling pathway ${ }^{29}$. This implied that NLR-mediated cell death also might be connected with EDS1/PAD4 pathway. Salicylic acid (SA) may play an important role in the thermoregulation of plant NLR-mediated cell death ${ }^{30}$.

Leaf senescence is one of the programmed cell death (PCD) and is regulated by ethylene (ET) hormone 32. Although SA regulates key pathways of plant cell death and immunity, this regulation is inhibited by ET ${ }^{30}$. Interestingly, ET did not affect leaf senescence in young seedlings ${ }^{33}$. There is a sufficient possibility that the newly generated upper leaves can escape the effects of ET when used for agroinfiltration. Arabidopsis onset of leaf death (old) mutants is allelic to CONSTITUTIVE EXPRESSER OF PR GENE5 (CPR5), which showed senescence symptoms in young seedlings ${ }^{34,35}$. Interestingly, CPR5 maintains the steady state level of nicotinamide adenine dinucleotide (NAD) ${ }^{36}$, suggesting that NAD homeostasis also may affect NLR-mediated cell death including autoimmune activity. CPR5 associated with a novel nucleoporin PLANT NUCLEAR ENVELOPE TRANSMEMBRANE 1 (PNET1) ${ }^{37}$. Function of human PNET1 homolog is important to the cell cycle regulation ${ }^{38}$. This suggests that CPR5-PNET1 may have a dual function between cell cycle and immune pathways in the nuclear pore. Thus, leaf positiondependent HR cell death might be associated with cell cycle and immunity.

\section{Conclusions}

We proposed optimized transient HR cell death assay conditions for NLR studies using tobacco plant. When temperature, humidity and leaf position conditions are optimally adjusted, various NLR-mediated effector triggered HR cell death and autoimmune phenotypes can be observed. 


\section{Methods}

Plant Materials and Growth Conditions

Nicotiana tabacum cv. Petite Gerard and Nicotiana benthamiana plants were sown on soil and grown at $22^{\circ} \mathrm{C}$ under long day condition ( $16 \mathrm{~h}$ light $/ 8 \mathrm{~h}$ dark) with $55 \%$ relative humidity.

Agrobacterium strains and vector constructions

Agrobacterium tumefaciens GV3101 was used in infiltration assays with tobacco leaves. The Agrobacterium strain GV3101 competent cells were thawed on ice and added $1 \mu \mathrm{L}$ recombined plant expression vector, then kept in ice for $5 \mathrm{~min}$. Then the mixture was fast frozen in liquid nitrogen for $5 \mathrm{~min}$, followed by an incubation at $37^{\circ} \mathrm{C}$ for $5 \mathrm{~min}$. After that, the mixture was kept in ice for $5 \mathrm{~min}$ and added 1 $\mathrm{mL}$ fresh Luria Broth (LB) liquid medium. After a culture in shaker for $1 \mathrm{~h}$ at $28{ }^{\circ} \mathrm{C}, 200 \mathrm{rpm}, 100 \mu \mathrm{L}$ cells were plated on a LB agar plate containing rifampicin $(25 \mathrm{mg} / \mathrm{L})$ and kanamycin $(100 \mathrm{mg} / \mathrm{L})$, and then cultured for 2 days at $28{ }^{\circ} \mathrm{C}$.

Briefly, genomic fragments of full-length RRS1-R and RRS1-S were PCR-amplified from Arabidopsis thaliana genomic DNA, accessions Ws-2 and Col-0. The genomic fragments of RRS1 were PCR-amplified with primers containing $4 \mathrm{bp}$ specific overhangs and $B$ sal recognition sequence and cloned into the pCR8/GW/TOPO (ThermoFisher). The resulting pCR8 constructs were subsequently used for Golden Gate assembly in plCH86988 ${ }^{39}$. C-cCFP, C-nCerulean, and N-cCFP tags were introduced into the RRS1 ${ }^{21}$. 35S::RPS4-HA, 35S:::RPS4-Myc, 35S::RRS1-R-HF, 35S::AvrRps4-GFP, 35S::AvrRps4 ${ }^{E 187 A}$-GFP,

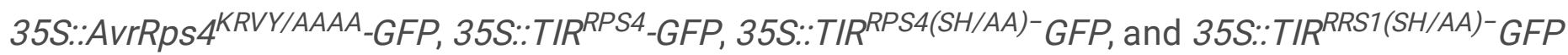
used in this study were described previously $15,16,17,21$.

Agrobacterium tumefaciens infiltration in tobacco plant

A single positive colony of Agrobacterium was inoculated in $3 \mathrm{~mL} \mathrm{LB}$ liquid medium ( $25 \mathrm{mg} / \mathrm{L}$ rifampicin and $100 \mathrm{mg} / \mathrm{L}$ kanamycin) and cultured for 1 day in a shaker at $28{ }^{\circ} \mathrm{C}, 200 \mathrm{rpm}$. Then, $100 \mu \mathrm{L}$ Agrobacterium cells were transferred into $5 \mathrm{~mL}$ fresh LB liquid medium supplemented with abovementioned antibiotics and cultured overnight at $28{ }^{\circ} \mathrm{C}, 200 \mathrm{rpm}$. The agrobacterium cells were pelleted by centrifugation (3000 rpm, $10 \mathrm{~min}$ ) and resuspended with $1 \mathrm{~mL}$ infiltration buffer (10 mM MgCl $2,10 \mathrm{mM}$ MES, pH 5.6). The re-suspended agro-cells were then diluted to $\mathrm{OD}_{600}=0.5$ with infiltration buffer. Fully expanded leaves of 4-5 weeks old tobacco plant were syringe-infiltrated. The leaf-infiltrated plants were dried with paper, and the humidity in the greenhouse was maintained at $55 \%$. After 1 day, the plants were transferred to growth chamber with a16-h light/8-h dark photoperiod at $22^{\circ} \mathrm{C}, 65 \%$ humidity or transferred to growth chamber at $22^{\circ} \mathrm{C}, 10 \%$ humidity. It is possible to test in a laboratory with a relative humidity of $10 \%$ and at $20-22^{\circ} \mathrm{C}$. The HR phenotype was confirmed at 3-5 dpi.

\section{Abbreviations}


NLR

Nucleotide-binding leucine-rich repeat (NLR) resistance $(R)$ proteins

HR

hypertensive response

T3SE

type three secreted system

ETI

effector-triggered immunity

ID

integrated domain

TIR

Toll-like, Interleukin-1 receptor, Resistance protein

$\mathrm{CC}$

coiled-coil

BiFC

Bimolecular fluorescence complementation

$\mathrm{CNL}$

CC-NLR

TNL

CC-NLR

NAD+

nicotinamide adenine dinucleotide

CADPR

non-canonical variant cyclic ADPR

WGS

whole genome sequencing

PCD

programmed cell death

CPR5

CONSTITUTIVE EXPRESSER OF PR GENE5

PNET1

PLANT NUCLEAR ENVELOPE TRANSMEMBRANE 1

\section{Declarations}

\section{Acknowledgements}

Author would like to thank Dr. Panagiotis F. Sarris for Golden Gate cloning the 35S::cCFP-RRS1-SnCerulean, 35S::cCFP-RRS1-R-nCerulean, and 35S::RRS1-R-cCFP.

\section{Funding}


This project was funded by the Next-Generation BioGreen 21 Program (Project No. PJ01365301) by Rural Development Administration and National Research Foundation of Korea (NRF) grant funded by the Korea government (MEST) (No.18A18297611), Republic of Korea.

\section{Author information}

Department of Biology, Kunsan National University, Gunsan 54150, Republic of Korea

Sung Un Huh

\section{Contributions}

S.U.H designed the research, performed the experiments, analyzed the data, wrote the read and approved the final manuscript.

\section{Availability of data and materials}

All data and material generated or analyzed during this study are included in this published article.

\section{Ethics approval and consent to participate}

Not applicable.

\section{Consent for publication}

Not applicable.

\section{Competing interests}

The authors declare that they have no competing interests.

\section{References}

1. Chaloner TM, Gurr SJ, Bebber DP. Plant pathogen infection risk tracks global crop yields under climate change. Nature Climate Change 2021, 11(8): 710-715.

2. Velasquez AC, Castroverde CDM, He SY. Plant-Pathogen Warfare under Changing Climate Conditions. Curr Biol 2018, 28(10): R619-R634.

3. McCann HC, Guttman DS. Evolution of the type III secretion system and its effectors in plant-microbe interactions. New Phytol 2008, 177(1):33-47.

4. Pruitt RN, Gust AA, Nurnberger T. Plant immunity unified. Nat Plants 2021, 7(4): 382-383.

5. Bentham A, Burdett $H$, Anderson PA, Williams SJ, Kobe B. Animal NLRs provide structural insights into plant NLR function. Ann Bot 2017, 119(5): 827-702. 
6. Adachi H, Derevnina L, Kamoun S. NLR singletons, pairs, and networks: evolution, assembly, and regulation of the intracellular immunoreceptor circuitry of plants. Curr Opin Plant Biol 2019, 50: 121131.

7. Narusaka M, Shirasu K, Noutoshi Y, Kubo Y, Shiraishi T, Iwabuchi M, et al. RRS1 and RPS4 provide a dual Resistance-gene system against fungal and bacterial pathogens. Plant J 2009, 60(2): 218-226.

8. Birker D, Heidrich K, Takahara H, Narusaka M, Deslandes L, Narusaka Y, et al. A locus conferring resistance to Colletotrichum higginsianum is shared by four geographically distinct Arabidopsis accessions. Plant J 2009, 60(4): 602-613.

9. Guo H, Ahn HK, Sklenar J, Huang J, Ma Y, Ding P, et al. Phosphorylation-Regulated Activation of the Arabidopsis RRS1-R/RPS4 Immune Receptor Complex Reveals Two Distinct Effector Recognition Mechanisms. Cell Host Microbe 2020, 27(5): 769-781 e766.

10. Menna A, Nguyen D, Guttman DS, Desveaux D. Elevated Temperature Differentially Influences Effector-Triggered Immunity Outputs in Arabidopsis. Front Plant Sci 2015, 6: 995.

11. Kiraly L, Hafez YM, Fodor J, Kiraly Z. Suppression of tobacco mosaic virus-induced hypersensitivetype necrotization in tobacco at high temperature is associated with downregulation of NADPH oxidase and superoxide and stimulation of dehydroascorbate reductase. J Gen Viro/ 2008, 89(Pt 3): 799-808.

12. Desaint H, Aoun N, Deslandes L, Vailleau F, Roux F, Berthome R. Fight hard or die trying: when plants face pathogens under heat stress. New Phytol 2021, 229(2): 712-734.

13. Cesari S, Kanzaki H, Fujiwara T, Bernoux M, Chalvon V, Kawano Y, et al. The NB-LRR proteins RGA4 and RGA5 interact functionally and physically to confer disease resistance. EMBO J 2014, 33(17): 1941-1959.

14. Maqbool A, Saitoh H, Franceschetti M, Stevenson CE, Uemura A, Kanzaki H, et al. Structural basis of pathogen recognition by an integrated HMA domain in a plant NLR immune receptor. Elife 2015, 4.

15. Huh SU, Cevik V, Ding P, Duxbury Z, Ma Y, Tomlinson L, et al. Protein-protein interactions in the RPS4/RRS1 immune receptor complex. PLoS Pathog 2017, 13(5): e1006376.

16. Williams SJ, Sohn KH, Wan L, Bernoux M, Sarris PF, Segonzac C, et al. Structural basis for assembly and function of a heterodimeric plant immune receptor. Science 2014, 344(6181): 299-303.

17. Sohn KH, Hughes RK, Piquerez SJ, Jones JD, Banfield MJ. Distinct regions of the Pseudomonas syringae coiled-coil effector AvrRps4 are required for activation of immunity. Proc Natl Acad Sci U SA 2012, 109(40): 16371-16376. 
18. Tasset C, Bernoux M, Jauneau A, Pouzet C, Briere C, Kieffer-Jacquinod S, et al. Autoacetylation of the Ralstonia solanacearum effector PopP2 targets a lysine residue essential for RRS1-R-mediated immunity in Arabidopsis. PLoS Pathog 2010, 6(11): e1001202.

19. Grund E, Tremousaygue D, Deslandes L. Plant NLRs with Integrated Domains: Unity Makes Strength. Plant Physiol 2019, 179(4): 1227-1235.

20. Ma Y, Guo H, Hu L, Martinez PP, Moschou PN, Cevik V, et al. Distinct modes of derepression of an Arabidopsis immune receptor complex by two different bacterial effectors. Proc Natl Acad Sci U SA 2018, 115(41): 10218-10227.

21. Sarris PF, Duxbury Z, Huh SU, Ma Y, Segonzac C, Sklenar J, et al. A Plant Immune Receptor Detects Pathogen Effectors that Target WRKY Transcription Factors. Cell 2015, 161(5): 1089-1100.

22. Maruta N, Burdett H, Lim BYJ, Hu X, Desa S, Manik MK, et al. Structural basis of NLR activation and innate immune signalling in plants. Immunogenetics 2022.

23. Essuman K, Summers DW, Sasaki Y, Mao X, Yim AKY, DiAntonio A, et al. TIR Domain Proteins Are an Ancient Family of NAD(+)-Consuming Enzymes. Curr Bio/ 2018, 28(3): 421-430 e424.

24. Horsefield S, Burdett $H$, Zhang X, Manik MK, Shi Y, Chen J, et al. NAD (+) cleavage activity by animal and plant TIR domains in cell death pathways. Science 2019, 365(6455): 793-799.

25. Wan L, Essuman K, Anderson RG, Sasaki Y, Monteiro F, Chung EH, et al. TIR domains of plant immune receptors are $\mathrm{NAD}(+)$-cleaving enzymes that promote cell death. Science 2019, 365(6455): 799-803.

26. Li Q, Jiang XM, Shao ZQ. Genome-Wide Analysis of NLR Disease Resistance Genes in an Updated Reference Genome of Barley. Front Genet 2021, 12: 694682.

27. Wang L, Zhao L, Zhang X, Zhang Q, Jia Y, Wang G, et al. Large-scale identification and functional analysis of NLR genes in blast resistance in the Tetep rice genome sequence. Proc Natl Acad Sci US A 2019, 116(37): 18479-18487.

28. Van de Weyer AL, Monteiro F, Furzer OJ, Nishimura MT, Cevik V, Witek K, et al. A Species-Wide Inventory of NLR Genes and Alleles in Arabidopsis thaliana. Cell 2019, 178(5): 1260-1272 e1214.

29. Disch EM, Tong M, Kotur T, Koch G, Wolf CA, Li X, et al. Membrane-Associated Ubiquitin Ligase SAUL1 Suppresses Temperature- and Humidity-Dependent Autoimmunity in Arabidopsis. Mol Plant Microbe Interact 2016, 29(1): 69-80.

30. Li Z, Liu H, Ding Z, Yan J, Yu H, Pan R, et al. Low Temperature Enhances Plant Immunity via Salicylic Acid Pathway Genes That Are Repressed by Ethylene. Plant Physiol 2020, 182(1): 626-639. 
31. Zhang Y, Goritschnig S, Dong X, Li X. A gain-of-function mutation in a plant disease resistance gene leads to constitutive activation of downstream signal transduction pathways in suppressor of npr1-1, constitutive 1. Plant Cel/ 2003, 15(11): 2636-2646.

32. Koyama T. A hidden link between leaf development and senescence. Plant Sci 2018, 276: 105-110.

33. Jing HC, Schippers JH, Hille J, Dijkwel PP. Ethylene-induced leaf senescence depends on age-related changes and OLD genes in Arabidopsis. J Exp Bot 2005, 56(421): 2915-2923.

34. Jing HC, Dijkwel PP. CPR5: A Jack of all trades in plants. Plant Signal Behav 2008, 3(8): 562-563.

35. Jing HC, Hebeler R, Oeljeklaus S, Sitek B, Stuhler K, Meyer HE, et al. Early leaf senescence is associated with an altered cellular redox balance in Arabidopsis cpr5/old1 mutants. Plant Biol (Stuttg) 2008, 10 Suppl 1: 85-98.

36. Schippers JH, Nunes-Nesi A, Apetrei R, Hille J, Fernie AR, Dijkwel PP. The Arabidopsis onset of leaf death 5 mutation of quinolinate synthase affects nicotinamide adenine dinucleotide biosynthesis and causes early ageing. Plant Cell 2008, 20(10):2909-2925.

37. Tang Y, Huang A, Gu Y. Global profiling of plant nuclear membrane proteome in Arabidopsis. Nat Plants 2020, 6(7): 838-847.

38. Fujitomo T, Daigo Y, Matsuda K, Ueda K, Nakamura Y. Critical function for nuclear envelope protein TMEM209 in human pulmonary carcinogenesis. Cancer Res 2012, 72(16): 4110-4118.

39. Weber E, Engler C, Gruetzner R, Werner S, Marillonnet S. A modular cloning system for standardized assembly of multigene constructs. PLoS One 2011, 6(2): e16765.

\section{Figures}


A

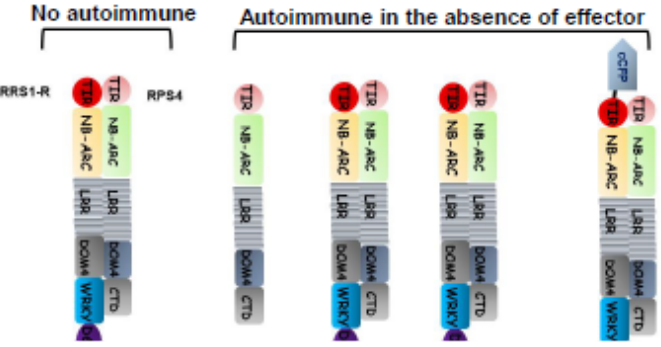

D

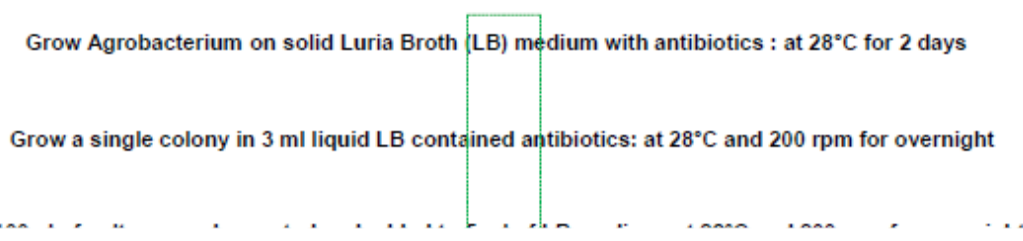

\section{Figure 1}

\section{Three HR cell death systems utilizing the paired NLR RPS4/RRS1}

A. The helper RPS4 autoimmune activity is suppressed by sensor RRS1. In the absence of RRS1, RPS4 exhibits autoimmune activity. When cCFP or nCerulean is fused to N-or C- of RRS1, it causes structural modification of RRS1. Thus, the ability of RRS1 to inhibit RPS4 autoimmune activity is lost. In the absence of effectors, the autoimmune activity of paired NLRs can be assessed. B. TIR domain of helper RPS4 is capable of generating an autoimmune signal by itself. Oligomerization of TIR RPS4 is important for generating an HR cell death signal. This TIR ${ }^{R P S 4}$-dependent HR can be repressed by coexpressing of TIR domain of RRS1. C. RPS4/RRS1 directly recognizes effectors and induces NLR-mediated HR cell death. Sensor RRS1 has an integration domain (ID) WRKY and is currently known to recognize three different pathogen effectors: AvrRps4 (Pseudomonas syringae), PopP2 (Ralstonia solanacearum), and an unknown effector (Colletotrichum higginsianum). D. Schematic of Agrobacterium-mediated transient expression for HR cell death assay. 
A

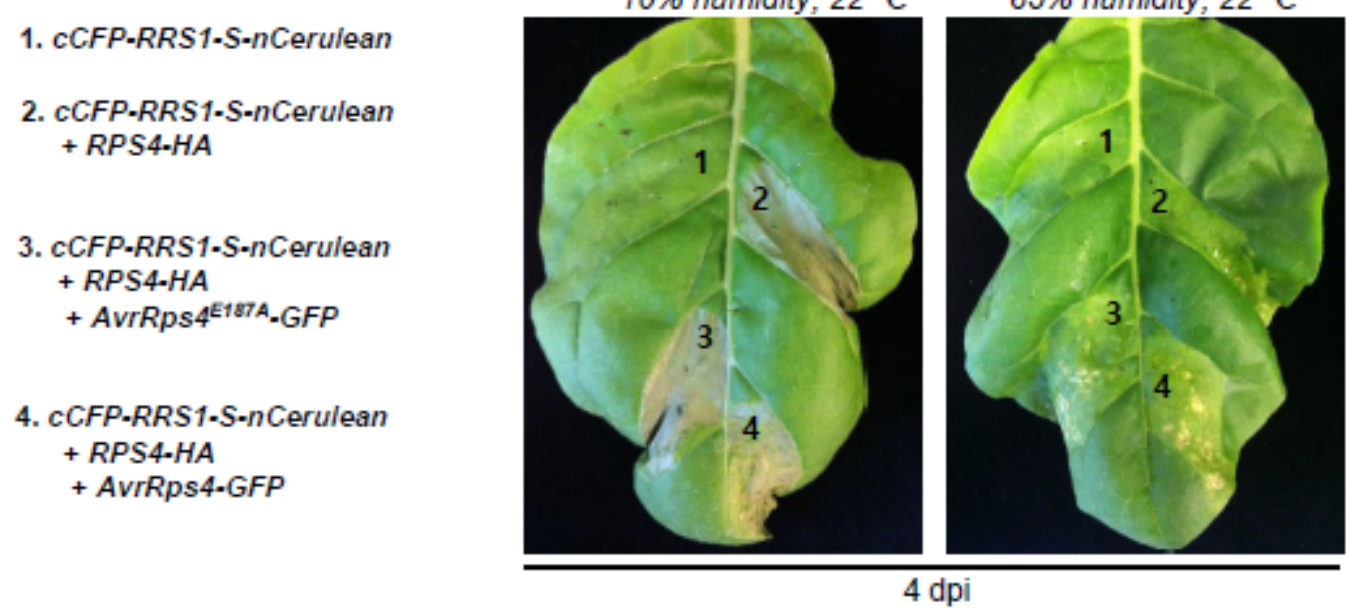

B
1. RRS1-R-nCerulean
2. RRS1-R-nCerulean + RPS4-myc
3. RRS1-R-CCFP
4. RRS1-R-cCFP + RPS4-myc
5. RRS1-R-HF
6. $R R S 1-R \cdot H F$
+ RPS4-myc

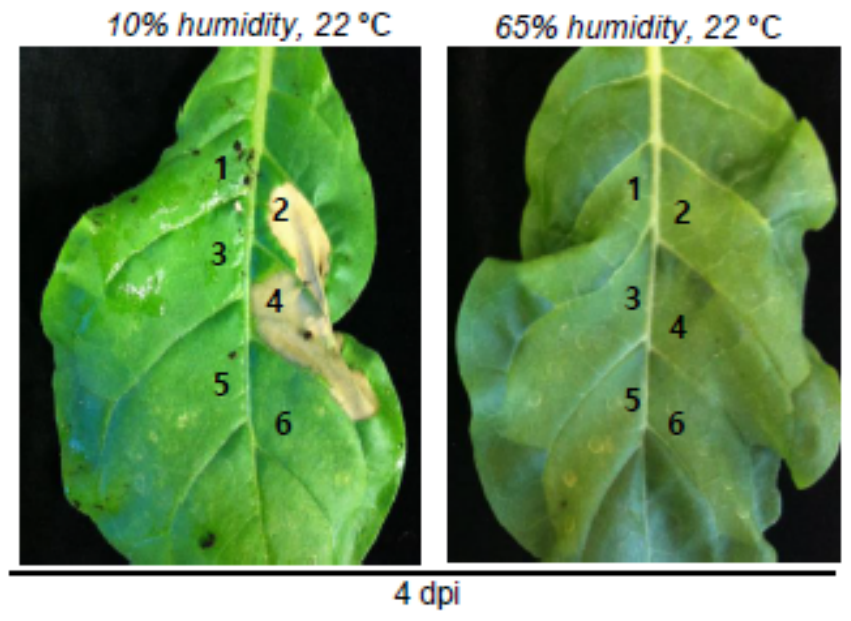

\section{Figure 2}

\section{RPS4/RRS1 autoimmune activity is enhanced by low humidity}

A. cCFP-RRS1S-nCerulean triggered RPS4-depentent autoimmune cell death in N. tabacum. 35S::cCFPRRS1-S-nCerulean or 35S::cCFP-RRS1-S-nCerulean/35S::RPS4-HA or 35S::cCFP-RRS1-SnCerulean/35S::RPS4-HA/35S::AvrRps4-GFP or 35S::cCFP-RRS1-S-nCerulean/35S::RPS4HA/35S::AvrRps4 ${ }^{E 187 A}$-GFP were transiently co-expressed in $N$. tabacum leaves. After agro-infiltration, the tobacco plants stayed at $55 \%$ humidity and $22{ }^{\circ} \mathrm{C}$ for 1 day. After 1 day, the tobacco plants were transferred to $10 \%$ and $65 \%$ humidity conditions, respectively. The HR cell death was confirmed on the 4 days. B. RRS1-R-nCerulean and RRS1-R-nCFP triggered RPS4-depentent autoimmune cell death in $N$. tabacum. 35S::RRS1-R-nCerulean or 35S::RRS1-R-nCerulean/35S::RPS4-Myc or 35S::RRS1-R-cCFP or 35S::RRS1-R-cCFP/35S::RPS4-Myc or 35S::RRS1-R-HF or 35S::RRS1-R-HF/35S::RPS4-Myc were transiently co-expressed in N. tabacum leaves. After agro-infiltration, the tobacco plants stayed at 55\% humidity and 
$22{ }^{\circ} \mathrm{C}$ for 1 day. After 1 day, the tobacco plants were transferred to $10 \%$ and $65 \%$ humidity conditions, respectively. The HR cell death was confirmed on the 4 days.

A

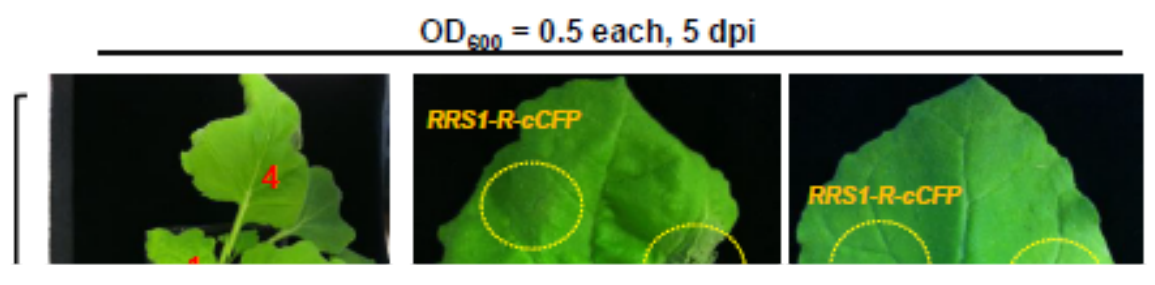

\section{Figure 3}

\section{Effect of leaf position and humidity on RPS4/RRS1-mediated autoimmunity in N. benthamiana}

A. The autoimmune activity of RPS4-mediated RRS1-cCFP or RRS1-nCerulean at leaf positions \#4 and \#5 was different at low humidity. The positions of the leaves are numbered from top to bottom. 35S::RRS1-RCCFP or 35S::RRS1-R-cCFP/35S::RPS4-Myc or 35S::RRS1-R-nCerulean or 35S:::RRS1-R-

$n$ Cerulean/35S::RPS4-Myc were transiently co-expressed in $N$. benthamiana leaf positions \#4 and \#5 at $55 \%$ humidity and $22{ }^{\circ} \mathrm{C}$ for 1 day. After 1 day, the tobacco plants were transferred to $10 \%$ humidity condition. The HR cell death was confirmed on the 5 days. B. The autoimmune activity of RPS4-mediated RRS1-CCFP or RRS1-nCerulean at leaf positions \#4 and \#5 was suppressed by high humidity. 35S::RRS1$R$-cCFP/35S::RPS4-Myc or 35S::RRS1-R-nCerulean/35S:::RPS4-Myc were transiently co-expressed in $N$. 
benthamiana leaf positions \#4 and \#5 at 55\% humidity and $22^{\circ} \mathrm{C}$ for 1 day. After 1 day, the tobacco plants were transferred to $65 \%$ humidity condition. The HR cell death was confirmed on the 5 days.

\section{Figure 4}

\section{Effect of leaf position on TIR RPS4-mediated autoimmune and RPS4/RRS1-mediated effector triggerred HR cell death in tobacco plants}

A. TIR RPS4-dependent autoimmune activity is different for each leaf position. $N$. benthamiana leaf was transiently agro-infiltrated with $35 S$ :::TIR ${ }^{R P S 4}$-GFP/35S.:GFP or inactive mutant $35 S::$ TIR $R^{R P S 4(S H / A A)}$ GFP/35S:::GFP or 35S::TIRRRS1_GFP/35S::GFP or 35S:::TIR RPS4(SH/AA)_GFP/inactive mutant

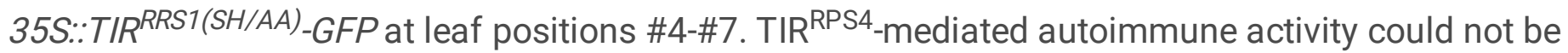
confirmed at leaf positions \#6-\#7, and the HR cell death for leaf positions \#4-\#5 was enlarged. The HR cell death was confirmed on the 3 days. B. RPS4/RRS1-mediated effector triggered HR cell death exhibited differently infiltrated leaf position in N. benthamiana. Agro-infiltration was performed with transiently coexpressed 35S::RRS1-R-HF/35S::RPS4-Myc with 35S::GFP or 35S::AvrRps4-GFP or nonfunctional mutant 35S::AvrRps4 ${ }^{K R V Y / A A A A}-G F P$ at leaf positions \#4-\#7 in N. benthamiana. RPS4/RRS1mediated HR cell death upon AvrRps4 perception could not be confirmed at leaf positions \#6-\#7, and the HR cell death for leaf positions \#4-\#5 was enlarged. The HR cell death was confirmed on the 3 days. C. RPS4/RRS1-mediated effector triggered HR cell death exhibited differently infiltrated leaf position in $N$. tabacum. Agro-infiltration was performed with transiently coexpressed 35S::RRS1-R-HF/35S::RPS4-Myc with 35S::GFP or 35S::AvrRps4-GFP ornon-functional mutant 35S::AvrRps4 ${ }^{K R V Y / A A A A}$-GFP at leaf positions \#4-\#5 in N. tabacum. The HR cell death for leaf positions \#4-\#5 was enlarged. The HR cell death was confirmed on the 4 days. 


\section{HR cell death intensity}

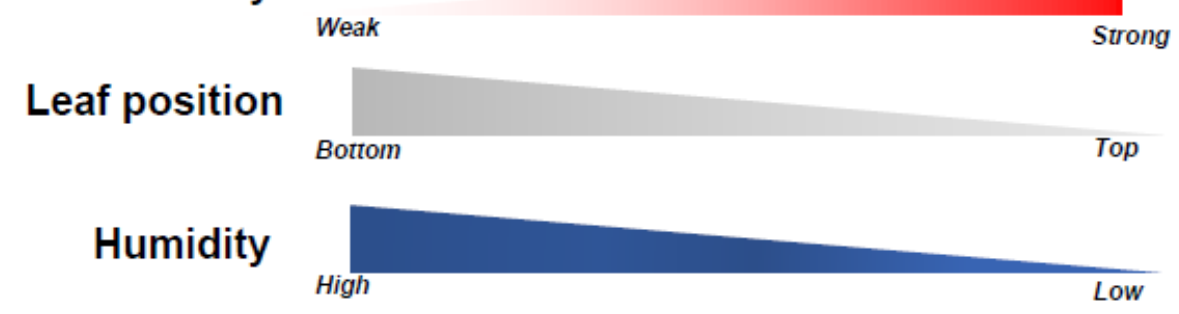

\section{Leaf senescence}

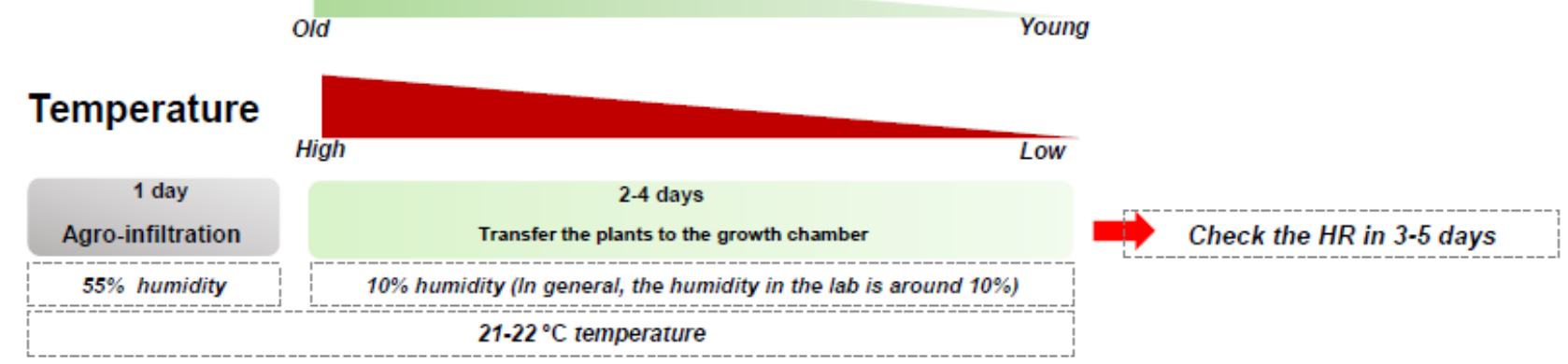

Figure 5

\section{NLR-mediated HR cell death intensity depends on leaf position and humidity}

The fastest and most accurate characterization of plant NLRs is the transient HR cell death assay using tobacco. Of course, NLRs that form very strong apoptosis signals can ignore various environmental conditions and confirm a phenotype of apoptosis. However, in HR cell death analysis using various NLR autoimmunity and effector recognition, cell death related to NLR function can be clearly identified when a low humidity of about $10 \%$ is used. Humidity conditions seem to behave very similarly to temperature conditions. High temperatures are often accompanied by high humidity. Therefore, it is known that the temperature condition is also a very important factor. Additionally, the leaf position of tobacco used for infiltration is considered to be another important factor. It is not yet known what kind of regulatory action leads to HR cell death, but it is thought to be somewhat related to leaf aging. As a result, it is believed that the assays to confirm HR cell death under normal plant culture conditions are affected by various environmental factors. 